\title{
Regional cortical thickness and neuroticism across the lifespan
}

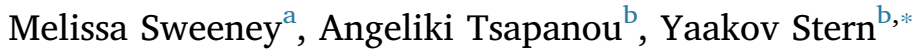 \\ a Teachers College, Columbia University, New York, NY, USA \\ ${ }^{\mathrm{b}}$ Cognitive Neuroscience Division, Department of Neurology and the Taub Institute, Columbia University College of Physicians and Surgeons, $630 \mathrm{~W} 168 \mathrm{th}$ Street, New \\ York, NY 10032, USA
}

\section{A R T I C L E I N F O}

\section{Keywords:}

Aging

Gyrus cinguli

Magnetic resonance imaging

Personality

Psychopathology

\begin{abstract}
A B S T R A C T
Neuroticism is associated with greater reactivity to stress and lifetime psychopathology. In the present study we examined the association between neuroticism and regional and total cortical thickness (CT) across the lifespan, accounting for gender. We also assessed interactions among these factors. 450 subjects between 19 and 80 years were included. Participants completed the International Personality Item Pool and a structural MRI scan. Total CT and the mean values of CT in five regions of interest were examined. We also investigated the interaction effect among age, gender and neuroticism on CT. There was no significant association between neuroticism and regional/total CT. A significant interaction between neuroticism, age, and gender on the thickness of the anterior cingulate was found. Women high in neuroticism showed a thinner anterior cingulate cortex than women low in neuroticism, with increasing age. In contrast, men high in neuroticism had a thicker anterior cingulate cortex compared to men low in neuroticism, with increasing age. Overall, high neuroticism was associated with differential cortical thickness in the anterior cingulate among men and women with increasing age.
\end{abstract}

\section{Introduction}

Neuroticism is the only personality trait that has been found to be highly associated with future development of psychopathology (Jeronimus et al., 2016; Krueger et al., 1996). It is characterized by emotional instability, self-consciousness, anxiety, negative affect, and sensitivity to negative environmental stimuli (Izard et al., 1993; Larsen and Ketelaar, 1991; Rusting and Larsen, 1997). Disorders commonly associated with high neuroticism include depression, anxiety, eating disorders, substance abuse, schizophrenia, and Alzheimer's Disease (AD) (Clark et al., 1994; Davis, 1997; Jylhä and Isometsä, 2006; Kilbey et al., 1992; Ormel et al., 2004; Prescott et al., 1997; Van Os and Jones, 2001; Wilson et al., 2006).

The literature on brain imaging of neuroticism and aging has been scarce. One twin study also showed that differences in levels of neuroticism associated with genetics become more substantial with age (Eaves and Eysenck, 1976).

Decreasing cortical thickness (CT) occurs during normal aging in the prefrontal cortex, but there are regional and age-related differences in heritability of cortical thickness (DeCarli et al., 1994; Jernigan et al., 2001; Lenroot et al., 2009; Raz et al., 1997; Sowell et al., 2003). Studies of the association between neuroticism and cortical thickness (CT) in specific brain regions have shown mixed results. A positive correlation was found between neuroticism and CT in the left parietal lobe in young women (Privado et al., 2017). In contrast, a study with a larger sample of adults over 20 years old of both genders found no relationship between neuroticism and CT (Bjørnebekk et al., 2013).

Additional studies point out the need to consider factors such as gender and age when investigating the relationship between neuroticism and regional CT. Wright et al. (2006) initially found an inverse relationship between neuroticism and orbitofrontal CT, but when gender was included as a covariate, these differences became non-significant. Another study found neuroticism was positively correlated with CT in the subgenual anterior cingulate cortex in females whereas the relationship was negative in males (Blankstein et al., 2009).

Other studies suggest that there could be differences in the relationship between neuroticism and regional CT as a function of age. In young subjects, neuroticism was found to be associated with thinner left orbitofrontal cortex (Wright et al., 2006), while in another study of elders, an association between neuroticism and thinner right superior frontal cortex and inferior frontal cortex was found (Wright et al., 2007). There have not yet been any studies comparing young and elderly subjects in terms of neuroticism and regional cortical thickness. Given that neuroticism remains stable throughout the lifespan, since it is a personality trait, its possible role in changes in brain morphology is lacking investigation, especially because it has been shown to be related

\footnotetext{
* Corresponding author.

E-mail address: ys11@cumc.columbia.edu (Y. Stern).
} 
to differences in CT and rates of future development of psychopathology.

There is currently no existing research on interactions among neuroticism, age, and gender on regional cortical thickness. Further research including a broader age range and taking account of gender, could better clarify the influence of these variables on the relationship between neuroticism and regional CT. Additionally, since CT may be affected by aging, investigation into differences across the lifespan may offer insight into cumulative effects of personality differences over the lifespan. The potentially complex interplay of these factors could help elucidate underlying etiology of many neurological and psychological disorders.

Thus, the aim of the present study was to examine the relationship between self-reported neuroticism and regional/total CT, in a large sample of adults, across the lifespan. Neuroticism was used as a predictor of CT since personality traits are stable throughout the lifespan, thus it could hold implications for morphological differences in aging or vulnerability to various neurological and psychiatric disorders. We further investigated this relationship by examining the potential interactions among the influence of age, gender, and neuroticism on regional CT.

\section{Methods}

\subsection{Participants}

The participating pool was from two large ongoing studies, the Reference Ability Neural Network (RANN) study and the Cognitive Reserve (CR) study. The RANN study examines the neural basis of agerelated changes in four cognitive abilities. The CR study investigates differences in cognitive processes or neural networks that allow some people with brain damage to perform better than others. More details can be found in previous publications (Habeck et al., 2016; Stern, 2009; Stern et al., 2014).

Four hundred and fifty subjects were included in the study. The demographics and characteristics of the sample are shown in Table 1. Participants were native English speakers, strongly right-handed, and had at least a fourth-grade reading level. They had a mean age of 53 with a standard deviation of 17 years and ranged from age 19 to 80 . The average length of education was 16 years with a standard deviation of 2.5 years. The sample was $55 \%$ female. Participants were recruited by market mailing or referrals from enrolled participants. Participants were included if they were English speakers, strongly right-handed according to the Edinburgh Handedness Index, and had at least a 4th grade reading level (WRAT-3 reading subtest), a score of at least 130 on the Mattis Dementia Rating Scale, and normal or corrected-to-normal vision (Lucas et al., 1998). Exclusion criteria included contraindications

Table 1

Demographic and clinical characteristics of our sample.

\begin{tabular}{ll}
\hline Characteristics & Total \\
\hline Total, $N$ & 450 \\
Age, mean (SD) & $53(17)$ \\
Range & $19-80$ \\
Education, years, mean (SD) & $16(2.5)$ \\
Females, $N$ (\%) & $247(55)$ \\
Race, $N$ (\%) & \\
Asian & $32(5.1)$ \\
Black/African American & $171(27.1)$ \\
Hispanic/Spanish & $1(0.2)$ \\
NA & $2(0.3)$ \\
Other & $61(9.6)$ \\
Pacific Islander & $5(0.8)$ \\
White & $363(57.1)$ \\
Anterior cingulate cortical Thickness, mean (SD) & $5.487(0.424)$ \\
Neuroticism, mean (SD) & $2.421(0.783)$
\end{tabular}

for MRI or any major medical, neurological, or psychiatric conditions. Thus, all participants had to be cognitively normal, without a diagnosis of Mild Cognitive Impairment (MCI) or dementia at baseline. The study was approved by the Institutional Review Board (IRB) of the College of Physicians and Surgeons of Columbia University. All participants provided written informed consent.

\subsection{Materials}

\subsubsection{Personality measurements}

The 50-item International Personality Item Pool (IPIP) was administered by self-report. The IPIP measures the Big-Five factors of extraversion, agreeableness, conscientiousness, neuroticism, and intellect/imagination (Donnellan et al., 2006). For each personality domain the scale ranged from 1 to 5 , with higher scores indicating higher levels of the trait. Reliability, as measured by Cronbach's alpha, is 0.864 for neuroticism (Socha et al., 2010). To create the graphs depicting the three-way interaction, those participants who scored above the mean (2.4) on the Neuroticism scale of the IPIP were considered "high neuroticism" and those with scores below the median were considered as "low neuroticism".

\subsection{Procedure}

\subsubsection{Structural MRI scan and image processing}

MRI images were acquired on a 3.0T Philips Achieva Magnet. Each scan used $240 \mathrm{~mm}$ field of view. The parameters for EPI acquisition were TE/TR (ms) 20/2000; Flip Angle $72^{\circ}$; In-plane resolution (voxels) $112 \times 112$; Slice thickness/gap (mm) 3/0; Slices 41. T1 scans for each participant were reconstructed with FreeSurfer (v5.1.0) software for human brain imaging analysis (http://surfer.nmr.mgh.harvard.edu). Cortical thickness in the regions was computed by standard FreeSurfer parcellation (Desikan et al., 2006). Reconstructions were checked, and if needed, manual control points and repeated reconstruction were used. CT values for regions, as well as the total CT were computed for each subject. FreeSurfer's subcortical segmentation and cortical parcellation has been shown to have comparable accuracy to manual labeling (Fischl et al., 2002, 2004).

\subsection{Statistical analysis}

All statistical analyses were performed using the Statistical Package for the Social Sciences (SPSS) 23 (SPSS, Chicago, Illinois). Nominally significant alpha values were defined as $p<0.05$. All of the variables were normally distributed, according to descriptive tests of asymmetry and kurtosis and visual inspection of histograms in SPSS.

Multiple linear regression models including neuroticism, age, gender, and their interactions were used to examine the association between each regional CT and neuroticism.

Based on the existing literature examining the relationship between neuroticism and regional CT, the following regions were included (a priori) in the analyses: frontal, temporal, parietal, anterior cingulate, and orbitofrontal (Bjørnebekk et al., 2013; Blankstein et al., 2009; Privado et al., 2017; Wright et al., 2007, 2006). Total CT was also included. Neuroticism, age, intracranial volume (ICV), education level (years), and regional and total CT were used as continuous variables.

\section{Results}

There were no significant associations between neuroticism and CT in the frontal $(B=-0.023, p=0.459)$, temporal $(B=-0.042$, $p=0.425)$, parietal $(B=-0.031, p=0.328)$, anterior cingulate $(B$ $=-0.002, p=0.930)$, or orbitofrontal $(B=-0.200, p=0.580)$ regions according to a multiple linear regression analysis which used neuroticism, age, gender, and their interactions to predict regional CT and total CT. The results for each region are shown in Table 2. The 
Table 2

Adjusted model (ICV, education).

\begin{tabular}{|c|c|c|c|c|}
\hline$\underline{\text { Region of interest }}$ & $\underline{\text { Neuroticism effect }}$ & $\underline{\text { Interaction Age x }}$ Neuroticism & $\underline{\text { Interaction Gender x Neuroticism }}$ & Interaction Age x Gender x Neuroticism \\
\hline Frontal & $\begin{array}{l}F=22.335, p=0.000 \\
B=-0.023, p=0.459\end{array}$ & $B=0.001, p=0.708$ & $B=-0.035, p=0.262$ & $B=0.000, p=0.882$ \\
\hline Temporal & $\begin{array}{l}F=20.049, p=0.000 \\
B=-0.042, p=0.425\end{array}$ & $B=-0.001, p=0.667$ & $B=0.011, p=0.829$ & $B=-0.002, p=0.580$ \\
\hline Parietal & $\begin{array}{l}F=9.300, p=0.000 \\
B=-0.031, p=0.328\end{array}$ & $B=0.001, p=0.694$ & $B=0.01, p=0.762$ & $B=0.000, p=0.895$ \\
\hline Orbitofrontal & $\begin{array}{l}F=14.530, p=0.000 \\
B=-0.200, p=0.580\end{array}$ & $B=-0.001, p=0.771$ & $B=-0.034, p=0.345$ & $B=0.000, p=0.820$ \\
\hline Anterior cingulate & $\begin{array}{l}F=3.566, p=0.001 \\
B=-0.002, p=0.930\end{array}$ & $B=-0.002, p=0.283$ & $B=0.036, p=0.199$ & $B=0.004, p=0.012$ \\
\hline Total CT & $\begin{array}{l}F=1.314, p=0.236 \\
B=0.006, p=0.723\end{array}$ & $B=0.000, p=0.673$ & $B=-0.002, p=0.901$ & $B=0.000, p=0.761$ \\
\hline
\end{tabular}

Significance level $p \leq .05$.

results were also non-significant for total CT $(B=0.006, p=0.723)$ (Table 2). This indicates that no significant relationship was found between neuroticism and the selected regions and total CT.

Next, we investigated the interaction effects for the relation of neuroticism, gender, and age to regional and total CT. Both the two-way interactions among age and neuroticism as well as gender and neuroticism were tested. The two-way interactions in the model were not significant in any brain region nor in total CT (Table 2). However, there was a significant interaction effect among age, gender, and neuroticism on anterior cingulate CT $(B=0.004, p=0.012)$. Table 2 shows the interaction among age, neuroticism, and anterior cingulate CT for males and for females. The presence of only a three-way interaction indicates that probably, the three variables act together on the anterior cingulate, but neither age nor gender alone interacts with neuroticism to produce any effect. The three-way interaction among age, gender, and neuroticism is such that women high in neuroticism had thinner cortex in the anterior cingulate with increasing age in comparison to women with low neuroticism. For men, those high in neuroticism had thicker cortex in the same area with increasing age, compared to men with low neuroticism.

The three-way interaction is shown in Fig. 1. The sample was split at the mean for age and neuroticism in order to visualize the three-way interaction by using two endpoints for each line depicting high or low neuroticism. No statistical analyses were done with the split sample.

In conclusion, our hypothesis for an effect of neuroticism on the selected brain regions and total CT was not confirmed; however, we did find a three-way interaction among neuroticism, age, and gender, as predicted $(p=0.012)$. Thus, the objective of the study, to examine the relationship among neuroticism and cortical thickness in several brain regions was met with the consideration of additional factors in their relationship.

\section{Discussion}

The current study investigated the associations between neuroticism and regional CT. There were no significant associations between neuroticism and total CT, or frontal, temporal, parietal, anterior cingulate, or orbitofrontal CT. There was a significant interaction between the effects of age, gender, and neuroticism on anterior cingulate CT. Analysis of the interaction effect indicates that high neuroticism in females is associated with a thinner anterior cingulate cortex with increasing age. In contrast, in males, high neuroticism was associated with a thicker anterior cingulate cortex as age increased. This suggests that neuroticism may moderate age-related differences on anterior cingulate $\mathrm{CT}$, depending on gender.

No significant associations between neuroticism and regional or total CT were found. Although the sample was large, the effect may be too small to have been detected in this sample. Alternatively, given the interaction between age, gender, and neuroticism, the effect of neuroticism on CT may be obscured by the opposing effects of gender and age. Since older men high in neuroticism had greater CT in the anterior cingulate than older women high in neuroticism, when the sample was not split by gender, the effects would be opposite and appear insignificant overall when the sample is combined.

The anterior cingulate is implicated in numerous psychological and neurological disorders so an interaction in this region may warrant further investigation due to its relevance to psychopathology (Cotter et al., 2001; Etkin et al., 2010; Pizzagalli et al., 2001; Rosenberg et al., 2004; Tekin et al., 2001). The region has previously been found to be associated with anxiety and mood disorders, with those higher in neuroticism showing greater activation in the region during emotional conflict (Haas et al., 2007). In addition, another study found late-onset depression to be associated with a thinner anterior cingulate cortex compared to controls (Lim et al., 2012). Lastly, a thinner cortex in the anterior cingulate has been found in individuals with $\mathrm{AD}$ in comparison with controls (Lerch et al., 2004). Such findings highlight the importance of this region in psychopathology and aging.

Numerous studies have found cortical thinning to occur during aging, but there is a pattern of thinning that spares certain brain regions (Fjell et al., 2009; Salat et al., 2004). In terms of the cingulate gyrus, the extant research is sparse and mixed. Two studies found thinning in the region, while another found thickening with age (Fjell et al., 2009; Salat et al., 2004; Thambisetty et al., 2010). The present study found that gender and level of neuroticism is related to the direction and magnitude of the CT difference across ages. Females high in neuroticism showed a thinner anterior cingulate cortex than females low in neuroticism with increasing age. Males high in neuroticism showed the opposite pattern, with a thicker cortex in this region with age while males low in neuroticism showed a thinner cortex with increasing age. Therefore, it appears that age-related differences in CT in the anterior cingulate might be at least partly driven by gender and level of neuroticism.

Gender, age, and neuroticism have all independently been found to be associated with various psychopathologies, but no studies have investigated the effect of an interaction among these factors on brain physiology. To our knowledge, there is also no existing research on interactions involving neuroticism on CT. One previous study found an interaction between neuroticism and age on cerebral white matter volume, with individuals higher in neuroticism having thinner cortex in this region with increasing age (Jackson et al., 2011). Although the present study focused on CT, interactions affecting white matter volume may be relevant since CT is one component of volume.

There are no existing studies examining interactions with gender on regional cortical thickness. However, a number of differences between the genders may account for the opposite pattern found between men and women in this study. The discrepancy may be partially explained by existing differences in CT between genders. Females appear to have a thicker cortex in numerous brain regions, with the cingulate gyrus 


\section{a. Interaction among neuroticism, gender, and age in males}

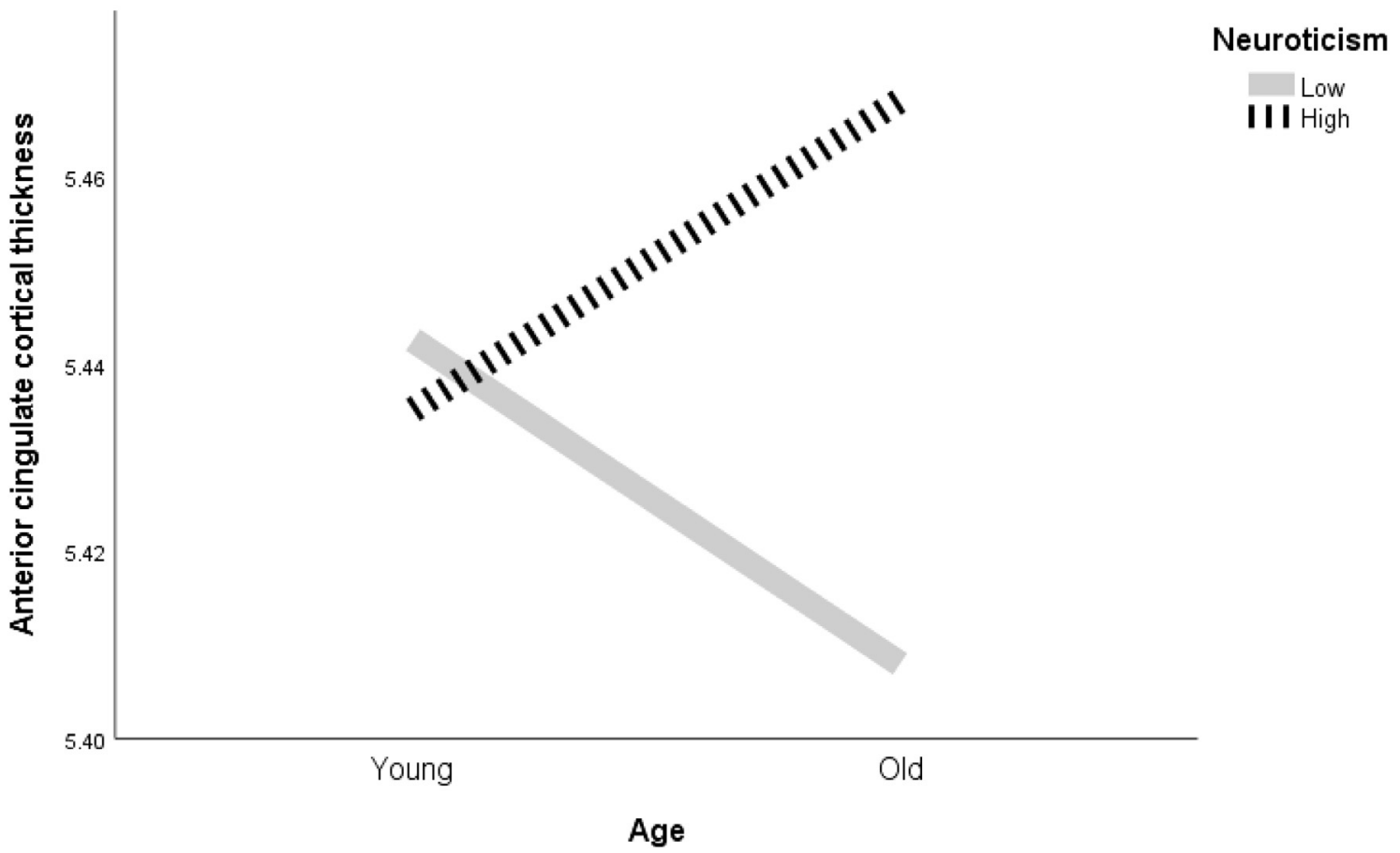

b. Interaction among neuroticism, gender, and age in females

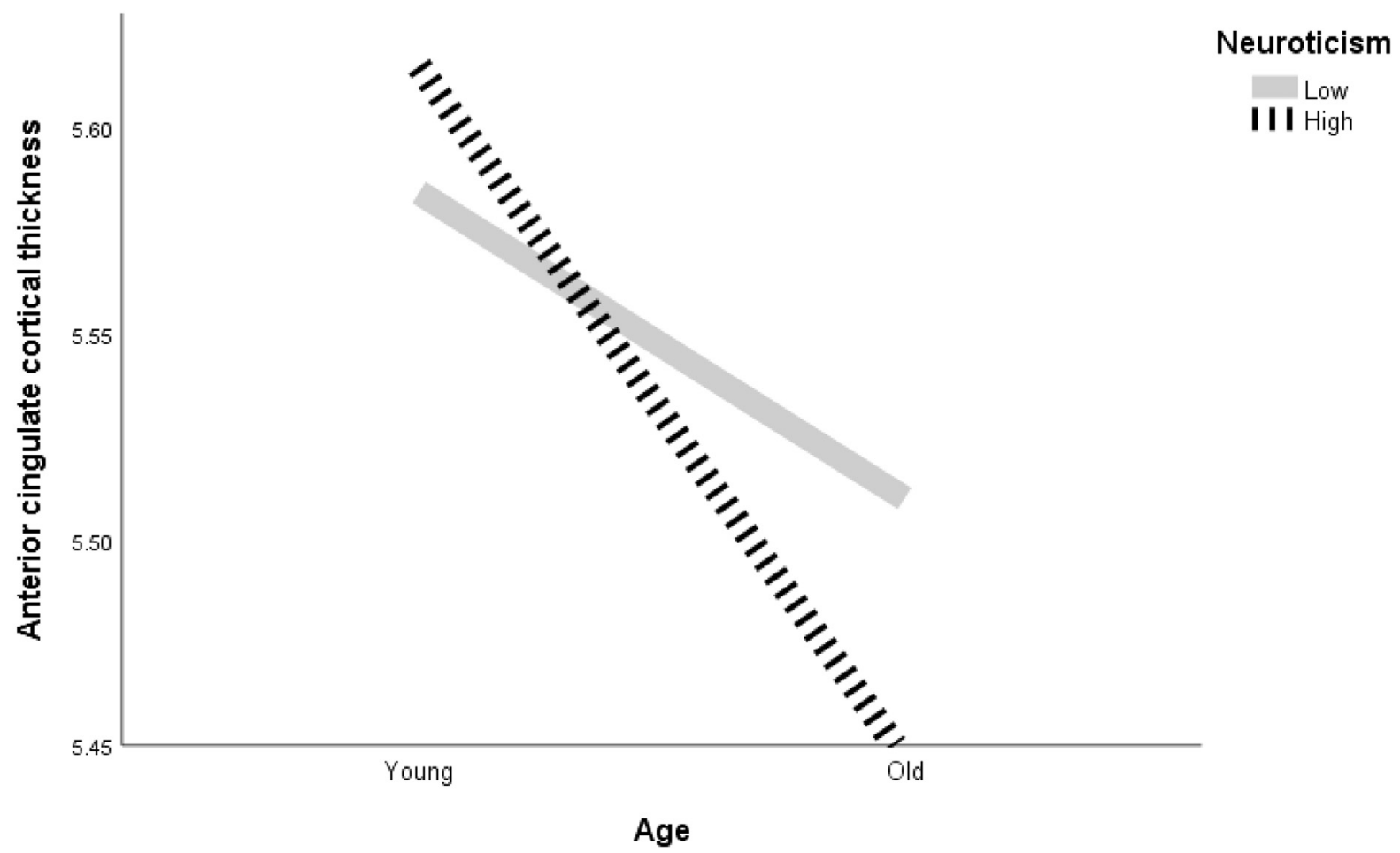

Fig. 1. (a) Interaction among neuroticism, gender, and age in males, (b) Interaction among neuroticism, gender, and age in females.

having one of the largest differences in thicknesses between genders (Luders et al., 2006; Sowell et al., 2006). This pre-existing difference in cortical thickness could impact the magnitude and direction of CT across age.

Finally, these results may also provide insight into gender differences in the prevalence of certain mental illnesses. Depression and anxiety disorders are more common in women, while substance use and suicide are more common in men (Baum and Grunberg, 1991; Boyd and Weissman, 1981; Canetto, 1991; Cleary, 1987). Since the effect of high neuroticism on CT in the anterior cingulate across ages affects men and women differently, it is possible that this is related to the differential rates of specific mental illnesses by gender. These results suggest that 
further investigation into the links among neuroticism, gender, age, and psychopathology may be warranted.

The current study has some limitations that should be noted. This study had a cross-sectional design so changes in CT during aging were not able to be investigated. There may also be cohort effects due to this design. For instance, one study showed that neuroticism scores increased between 1953 and 1993 (Twenge, 2000). These differences are hypothesized to relate to environmental and sociocultural factors, which could result in a cohort effect where some subjects of similar ages had different environmental experiences from other subjects that influence neuroticism scores (Twenge, 2000). Additionally, the IPIP is a self-reported measure.

This study, however, also has several strengths. The study's large sample size allowed for a more powerful analysis. It also had one of the largest age ranges among CT and neuroticism studies. Furthermore, it used more accurate imaging methods to investigate CT as a more specific measure of physiological differences. Lastly, it used a cognitively healthy group of participants.

Future research should focus on identifying additional brain regions that may be affected by neuroticism or its interaction with other factors. Lastly, longitudinal research on the relationship between the interaction among age, gender, and neuroticism with psychopathology could determine causation and direction of the effect.

In summary, the interaction between age, gender, and neuroticism in the anterior cingulate may have important implications for psychopathology research. It also could play a role in rectifying existing discrepancies in the literature on CT differences due to age and personality. It appears that there is a complex relationship among neuroticism, age, gender, and regional CT.

\section{Contributors}

M.S. and A.T. conceived of the presented idea. M.S. and Y.S. designed the study. M.S. conducted the analysis and interpretation. M.S. wrote the manuscript in consultation with A.T. and Y.S.

\section{Competing interests}

The authors declare no competing interests.

\section{Acknowledgments}

Funding: This research was funded by the National Institute on Aging (RO1AG038465 and ROIAG026158, both to Dr. Stern). The sponsors had no role in the study design, data collection, analysis, interpretation, writing of the report, or decision to submit the article for publication.

\section{References}

Baum, A., Grunberg, N.E., 1991. Gender, stress, and health. Health Psychol. 10, 80-85. Bjørnebekk, A., Fjell, A.M., Walhovd, K.B., Grydeland, H., Torgersen, S., Westlye, L.T., 2013. Neuronal correlates of the five factor model (FFM) of human personality: multimodal imaging in a large healthy sample. Neuroimage 65, 194-208.

Blankstein, U., Chen, J.Y., Mincic, A.M., McGrath, P.A., Davis, K.D., 2009. The complex minds of teenagers: neuroanatomy of personality differs between sexes. Neuropsychol 47, 599-603.

Boyd, J.H., Weissman, M.M., 1981. Epidemiology of affective disorders: a reexamination and future directions. Arch. Gen. Psychiatry 38, 1039-1046.

Canetto, S.S., 1991. Gender roles, suicide attempts, and substance abuse. J. Psychol. 125, 605-620.

Clark, L.A., Watson, D., Mineka, S., 1994. Temperament, personality, and the mood and anxiety disorders. J. Abnorm. Psychol. 103, 103.

Cleary, P.D., 1987. Gender differences in stress-related disorders. Gend. Stress 39-72.

Cotter, D., Mackay, D., Landau, S., Kerwin, R., Everall, I., 2001. Reduced glial cell density and neuronal size in the anterior cingulate cortex in major depressive disorder. Arch. Gen. Psychiatry 58, 545-553.

Davis, C., 1997. Normal and neurotic perfectionism in eating disorders: an interactive model. Int. J. Fat Disord. 22, 421-426.

DeCarli, C., Murphy, D., Gillette, J., Haxby, J., Teichberg, D., Schapiro, M., Horwitz, B.,
1994. Lack of age-related differences in temporal lobe volume of very healthy adults. Am. J. Neuroradiol. 15, 689-696.

Desikan, R.S., Ségonne, F., Fischl, B., Quinn, B.T., Dickerson, B.C., Blacker, D., Buckner, R.L., Dale, A.M., Maguire, R.P., Hyman, B.T., 2006. An automated labeling system for subdividing the human cerebral cortex on MRI scans into gyral based regions of interest. Neuroimage 31, 968-980.

Donnellan, M.B., Oswald, F.L., Baird, B.M., Lucas, R.E., 2006. The mini-IPIP scales: tinyyet-effective measures of the Big Five factors of personality. Psychological assessment 18,192

Eaves, L., Eysenck, H., 1976. Genotype x age interaction for neuroticism. Behav.Genet. 6 , 359-362.

Etkin, A., Prater, K.E., Hoeft, F., Menon, V., Schatzberg, A.F., 2010. Failure of anterior cingulate activation and connectivity with the amygdala during implicit regulation of emotional processing in generalized anxiety disorder. Am. J. Psychiatry 167, 545-554.

Fischl, B., Salat, D.H., Busa, E., Albert, M., Dieterich, M., Haselgrove, C., Van Der Kouwe, A., Killiany, R., Kennedy, D., Klaveness, S., 2002. Whole brain segmentation: automated labeling of neuroanatomical structures in the human brain. Neuron 33 , 341-355.

Fischl, B., Van Der Kouwe, A., Destrieux, C., Halgren, E., Ségonne, F., Salat, D.H., Busa, E., Seidman, L.J., Goldstein, J., Kennedy, D., 2004. Automatically parcellating the human cerebral cortex. Cereb. Cortex 14, 11-22.

Fjell, A.M., Westlye, L.T., Amlien, I., Espeseth, T., Reinvang, I., Raz, N., Agartz, I., Salat, D.H., Greve, D.N., Fischl, B., 2009. High consistency of regional cortical thinning in aging across multiple samples. Cereb. Cortex 19, 2001-2012.

Haas, B.W., Omura, K., Constable, R.T., Canli, T., 2007. Emotional conflict and neuroticism: personality-dependent activation in the amygdala and subgenual anterior cingulate. Behav. Neurosci. 121, 249.

Habeck, C., Gazes, Y., Razlighi, Q., Steffener, J., Brickman, A., Barulli, D., Salthouse, T. Stern, Y., 2016. The Reference Ability Neural Network Study: life-time stability of reference-ability neural networks derived from task maps of young adults. NeuroImage 125, 693-704.

Izard, C.E., Libero, D.Z., Putnam, P., Haynes, O.M., 1993. Stability of emotion experiences and their relations to traits of personality. J. Personal. Soc. Psychol. 64, 847.

Jackson, J., Balota, D.A., Head, D., 2011. Exploring the relationship between personality and regional brain volume in healthy aging. Neurobiol. Aging 32, 2162-2171.

Jernigan, T.L., Archibald, S.L., Fennema-Notestine, C., Gamst, A.C., Stout, J.C., Bonner, J., Hesselink, J.R., 2001. Effects of age on tissues and regions of the cerebrum and cerebellum. Neurobiol. Aging 22, 581-594.

Jeronimus, B., Kotov, R., Riese, H., Ormel, J., 2016. Neuroticism's prospective association with mental disorders halves after adjustment for baseline symptoms and psychiatric history, but the adjusted association hardly decays with time: a meta-analysis on 59 longitudinal/prospective studies with 443313 participants. Psychol. Med. 46, 2883-2906.

Jylhä, P., Isometsä, E., 2006. The relationship of neuroticism and extraversion to symptoms of anxiety and depression in the general population. Depress. Anxiety 23, 281-289.

Kilbey, M.M., Breslau, N., Andreski, P., 1992. Cocaine use and dependence in young adults: associated psychiatric disorders and personality traits. Drug Alcohol Depend. 29, 283-290.

Krueger, R.F., Caspi, A., Moffitt, T.E., Silva, P.A., McGee, R., 1996. Personality traits are differentially linked to mental disorders: a multitrait-multidiagnosis study of an adolescent birth cohort. J. Abnorm. Psychol. 105, 299.

Larsen, R.J., Ketelaar, T., 1991. Personality and susceptibility to positive and negative emotional states. J. Personal. Soc. Psychol. 61, 132.

Lenroot, R.K., Schmitt, J.E., Ordaz, S.J., Wallace, G.L., Neale, M.C., Lerch, J.P., Kendler, K.S., Evans, A.C., Giedd, J.N., 2009. Differences in genetic and environmental influences on the human cerebral cortex associated with development during childhood and adolescence. Hum. Brain Mapp. 30, 163-174.

Lerch, J.P., Pruessner, J.C., Zijdenbos, A., Hampel, H., Teipel, S.J., Evans, A.C., 2004. Focal decline of cortical thickness in Alzheimer's disease identified by computational neuroanatomy. Cereb. Cortex 15, 995-1001.

Lim, H.K., Jung, W.S., Ahn, K.J., Won, W.Y., Hahn, C., Lee, S.Y., Kim, I., Lee, C.U., 2012. Regional cortical thickness and subcortical volume changes are associated with cognitive impairments in the drug-naive patients with late-onset depression. Neuropsychopharmacology 37, 838-849.

Lucas, J.A., Ivnik, R.J., Smith, G.E., Bohac, D.L., Tangalos, E.G., Kokmen, E., GraffRadford, N.R., Petersen, R.C., 1998. Normative data for the Mattis dementia rating scale. J. Clin. Exp. Neuropsychol. 20, 536-547.

Luders, E., Narr, K.L., Thompson, P.M., Rex, D.E., Woods, R.P., DeLuca, H., Jancke, L., Toga, A.W., 2006. Gender effects on cortical thickness and the influence of scaling. Hum. Brain Mapp. 27, 314-324.

Ormel, J., Rosmalen, J., Farmer, A., 2004. Neuroticism: a non-informative marker of vulnerability to psychopathology. Soc. Psychiatry Psychiatr. Epidemiol. 39, 906-912.

Pizzagalli, D., Pascual-Marqui, R.D., Nitschke, J.B., Oakes, T.R., Larson, C.L., Abercrombie, H.C., Schaefer, S.M., Koger, J.V., Benca, R.M., Davidson, R.J., 2001. Anterior cingulate activity as a predictor of degree of treatment response in majo depression: evidence from brain electrical tomography analysis. Am. J. Psychiatry 158, 405-415.

Prescott, C.A., Neale, M.C., Corey, L.A., Kendler, K.S., 1997. Predictors of problem drinking and alcohol dependence in a population-based sample of female twins. J. Stud. Alcohol 58, 167-181.

Privado, J., Román, F.J., Saénz-Urturi, C., Burgaleta, M., Colom, R., 2017. Gray and white matter correlates of the Big Five personality traits. Neurosci 349, 174-184.

Raz, N., Gunning, F.M., Head, D., Dupuis, J.H., McQuain, J., Briggs, S.D., Loken, W.J., Thornton, A.E., Acker, J.D., 1997. Selective aging of the human cerebral cortex 
observed in vivo: differential vulnerability of the prefrontal gray matter. Cereb. cortex 7, 268-282.

Rosenberg, D.R., Mirza, Y., Russell, A., Tang, J., Smith, J.M., Banerjee, S.P., Bhandari, R., Rose, M., Ivey, J., Boyd, C., 2004. Reduced anterior cingulate glutamatergic concentrations in childhood OCD and major depression versus healthy controls. J. Am. Acad. Child Adolesc. Psychiatry 43, 1146-1153.

Rusting, C.L., Larsen, R.J., 1997. Extraversion, neuroticism, and susceptibility to positive and negative affect: a test of two theoretical models. Personal. Individ. Differ. 22, 607-612.

Salat, D.H., Buckner, R.L., Snyder, A.Z., Greve, D.N., Desikan, R.S., Busa, E., Morris, J.C., Dale, A.M., Fischl, B., 2004. Thinning of the cerebral cortex in aging. Cerebr. Cortex 14, 721-730.

Socha, A., Cooper, C.A., McCord, D.M., 2010. Confirmatory factor analysis of the M5-50: an implementation of the International Personality Item Pool item set. Psychol. Assess. 22, 43.

Sowell, E.R., Peterson, B.S., Kan, E., Woods, R.P., Yoshii, J., Bansal, R., Xu, D., Zhu, H., Thompson, P.M., Toga, A.W., 2006. Sex differences in cortical thickness mapped in 176 healthy individuals between 7 and 87 years of age. Cerebr. Cortex 17, 1550-1560.

Sowell, E.R., Peterson, B.S., Thompson, P.M., Welcome, S.E., Henkenius, A.L., Toga, A.W., 2003. Mapping cortical change across the human life span. Nat. Neurosci. 6, 309.

Stern, Y., 2009. Cognitive reserve. Neuropsychology 47, 2015-2028.
Stern, Y., Habeck, C., Steffener, J., Barulli, D., Gazes, Y., Razlighi, Q., Shaked, D., Salthouse, T., 2014. The Reference Ability Neural Network Study: motivation, design, and initial feasibility analyses. NeuroImage 103, 139-151.

Tekin, S., Mega, M.S., Masterman, D.M., Chow, T., Garakian, J., Vinters, H.V., Cummings, J.L., 2001. Orbitofrontal and anterior cingulate cortex neurofibrillary tangle burden is associated with agitation in Alzheimer disease. Ann. Neurol. 49, 355-361.

Thambisetty, M., Wan, J., Carass, A., An, Y., Prince, J.L., Resnick, S.M., 2010. Longitudinal changes in cortical thickness associated with normal aging. Neuroimage $52,1215-1223$.

Twenge, J.M., 2000. The age of anxiety? The birth cohort change in anxiety and neuroticism, 1952-1993. J. Personal. Soc. Psychol. 79, 1007-1021.

Van Os, J., Jones, P.B., 2001. Neuroticism as a risk factor for schizophrenia. Psychol. Med. 31, 1129-1134.

Wilson, R.S., Arnold, S.E., Schneider, J.A., Kelly, J.F., Tang, Y., Bennett, D.A., 2006. Chronic psychological distress and risk of Alzheimer's disease in old age. Neuroepidemiology 27, 143-153.

Wright, C.I., Feczko, E., Dickerson, B., Williams, D., 2007. Neuroanatomical correlates of personality in the elderly. Neuroimage 35, 263-272.

Wright, C.I., Williams, D., Feczko, E., Barrett, L.F., Dickerson, B.C., Schwartz, C.E., Wedig, M.M., 2006. Neuroanatomical correlates of extraversion and neuroticism. Cerebr. Cortex 16, 1809-1819. 